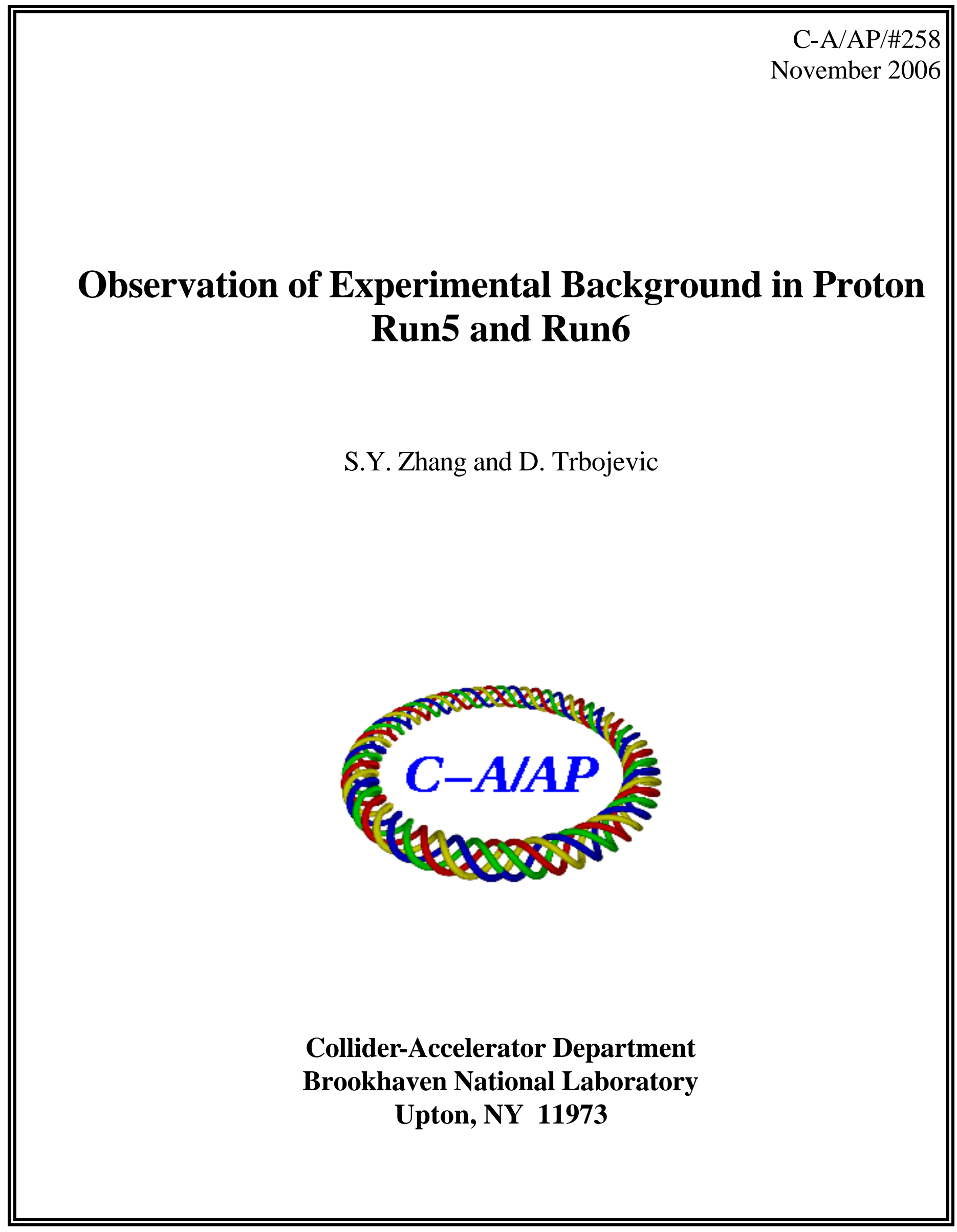




\title{
Observation of Experimental Background in Proton Run5 and Run6
}

\author{
S.Y. Zhang and D. Trbojevic
}

\begin{abstract}
The beam-gas has affected STAR background in Run5, but not in Run6. With much higher beam intensity in Run7, the beam-gas effect at STAR may be of concern. The ratio of ZDC background and coincident rate seems useful in proton runs in evaluating the experimental background.
\end{abstract}

\section{Introduction}

There are three main sources of the experimental background. The beamgas induced background is associated with the vacuum pressure, the beamchamber-interaction induced background can be improved by collimations, and the beam-beam induced background is somewhat inherent, and probably harmless for the experimental data taking.

The experimental background had affected STAR data taking in Run5, but not for PHENIX. In Run6, with significant improvement in STAR, the experimental background was not an important issue.

Observation shows that the beam-gas induced background was relevant for STAR in Run5. In Run6, the dynamic pressure rise at the STAR interaction region had significant improvements, and the beam-gas effect was reduced, but still identifiable. In Run7, the beam intensity will be $50 \%$ higher than in Run6, therefore, the beam-gas could be an issue.

The zero degree calorimeter (ZDC) is an essential luminosity detector for heavy ion operations in RHIC. In previous heavy ion runs, the ratio of ZDC background and ZDC coincident rate has been a useful indicator of the experimental background $[1,2]$. In proton Run5 and Run6, this ratio seems also useful. At STAR, this ratio was above 11 in Run5, but it was reduced to 
below 10 in Run6, probably due to the shieldings. On the other hand, this ratio at the PHENIX was below 10 for both Run5 and Run6, and PHENIX had no complain about the background.

In this article, we use background to represent the ZDC background (singles), and ZDC to represent ZDC coincident rate.

\section{Beam-gas background}

The beam-gas induced background is associated with the gas density at the interaction region (IR), and the beam intensity as well. The latter is essential for the machine luminosity, therefore, the gas density is the target to attack.

The static pressure at IR has limited to minimum in vacuum system design, however, once in a while, it could be raised to cause background problem. Usually, vacuum sublimations can be applied to mitigate. The background problem due to static pressure rising occurred in copper run 2005, and it is probably relevant for background complains at STAR in proton runs.

Beam induced dynamic pressure rise is more important in beam-gas background. At RHIC, the dynamic pressure rise for proton runs is mainly due to the electron cloud, where the relevant factors are the bunch spacing, bunch intensity, bunch length, and the chamber conditions.

In Fig.1, the beam intensity, pressure at IR6 (STAR), and the STAR background and ZDC of two typical fills each in proton Run5 and Run6 are shown for comparison.

The dynamic pressure rise in Run5 was not only higher than in Run6, but also lasted longer. It is clearly affected the STAR background for about an hour. The high background then affected ZDC. Note that the ZDC coincident rate can be contaminated, if the background singles is too high.

The bunch spacing of the fills in Run5 $(7325,7327)$ and Run6 $(7908,7909)$ are the same, $108 \mathrm{~ns}$. The bunch lengths are all around $8 \mathrm{~ns}$ (before the RF voltage ramping), and the IR6 vacuum chamber conditions are unchanged. For much higher bunch intensity $\left(1.3 \times 10^{11}\right.$ protons $)$ in Run6 than in Run5 $\left(0.9 \times 10^{11}\right.$ protons $)$, the dynamic pressure rise is lower. This pressure rise reduction is observed in Run6 for all warm section with unchanged chambers, and it is under investigation [3].

The details of the Fill 7908 in Run6 is shown in Fig.2, for the beam intensity, IR6 pressure, collimator positions, and the STAR background. At 

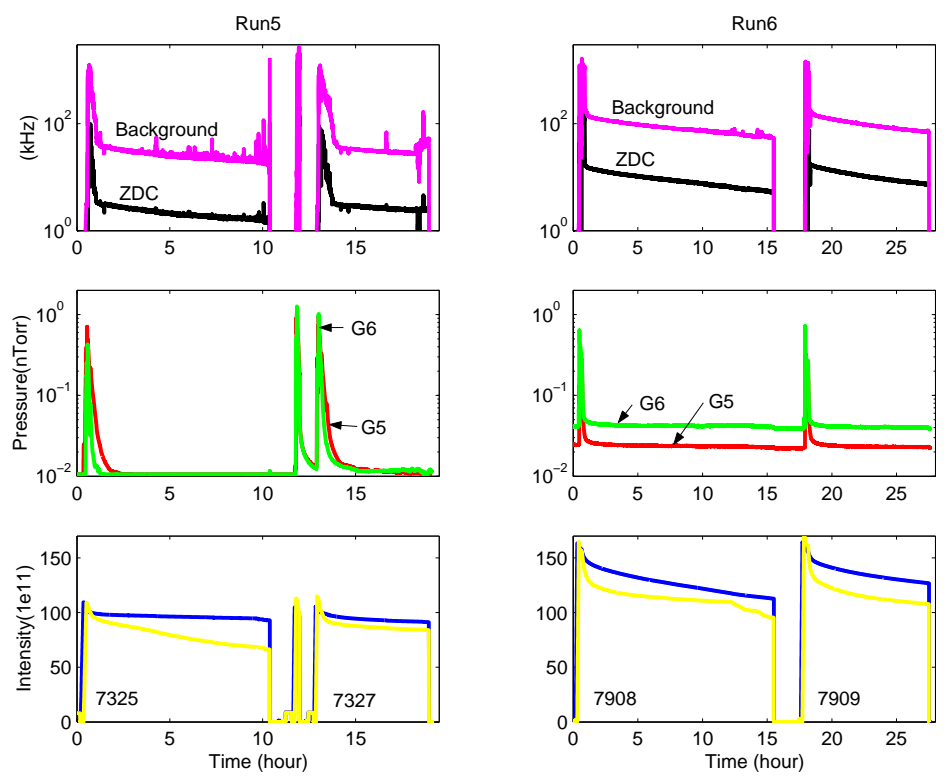

Figure 1: Beam intensity, pressure at STAR interaction region IR6, STAR background and ZDC are shown for typical fills in Run5 $(7325,7327)$ and Run6 (7908,7909).
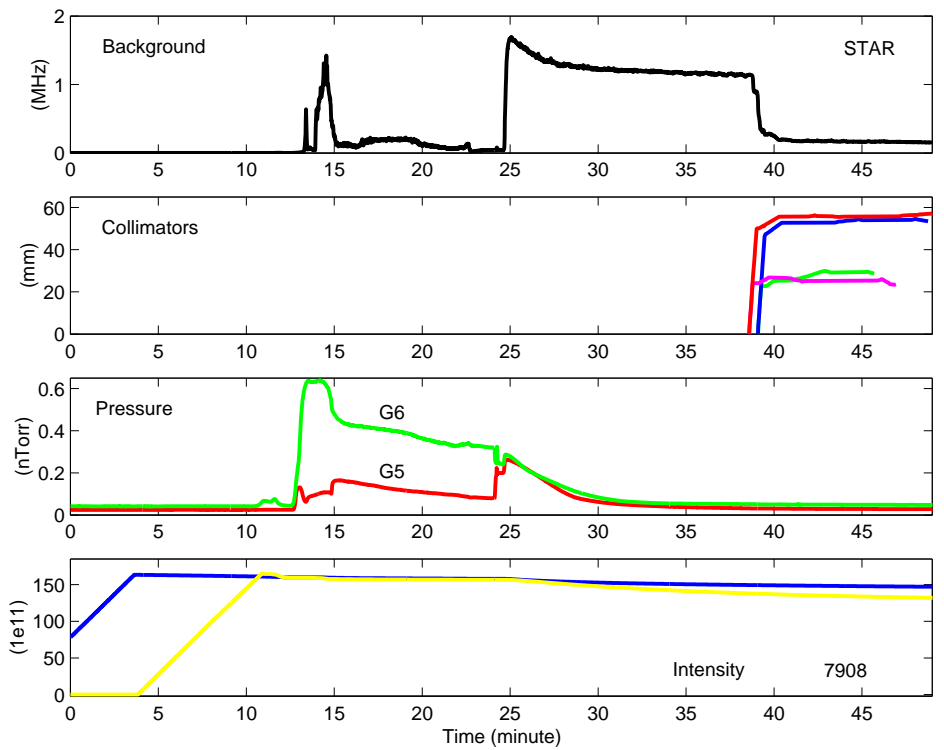

Figure 2: Beam intensity, pressure at IR6, collimator positions, and STAR background of Fill 7908 in Run6. 
25 minutes, the beam was put in collision, and at 49 minutes the collimation was applied. It can be seen that in this case the background was mainly reduced by the collimation. However, from 25 to 27 minutes, the beamgas effect can be identified from the pressure rise and the background. The related pressure rise was at about $10^{-10}$ to $3 \times 10^{-10}$ Torr.

In Run7, the bunch intensity will be $50 \%$ higher than Run6. The IR6 chamber will not change. The dynamic pressure rise could be much higher than in Run6, especially for shorter bunches, the beam-gas induced background might be of concern for STAR.

\section{$3 \quad$ ZDC background and coincident rate ratio}

The mechanism of the beam-beam created ZDC coincidence and singles is somewhat understood for heavy ions. To predict the ZDC coincident rate, the nuclear collision and mutual Coulomb dissociation cross sections, $\sigma_{n}$ and $\sigma_{m c}$, respectively, can be used. To predict the ZDC singles, the single Coulomb dissociation cross section, $\sigma_{s c}$, can be applied.

For example, for $\mathrm{Au}-\mathrm{Au}$ collisions, $\sigma_{n}+\sigma_{m c} \approx 6.1+4=10.1 \mathrm{~b}$, and $\sigma_{s c} \approx 92 b[1]$. The background/ZDC ratio of about 10 has been observed at store, whenever the beam-gas created background has been reduced and the collimation has applied. For $\mathrm{Cu}-\mathrm{Cu}$ collisions, $\sigma_{n}+\sigma_{m c} \approx 2.9+0=2.9$ $b$ and $\sigma_{s c} \approx 4.7 b[2]$, and the background/ZDC ratio of about 3 is observed (the background includes coincidence).

The mechanism for proton collisions are not well understood. However, the ratio of background and ZDC seems still useful as an indicator for the experimental background evaluation.

In Fig.3, the beam intensity, PHENIX and STAR background and ZDC are shown for the last 30 fills in Run5 and in Run6.

Typical ZDC for both PHENIX and STAR in Run5 is $3 \mathrm{kHz}$, and it is 10 $\mathrm{kHz}$ in Run6. It can be noticed that the typical ZDC background in Run6 is much higher than that in Run5, yet the experimental background was not a problem in Run6. This suggests that the background shown, during the long store periods, is mainly from beam-beam, which is inherent and harmless.

On the other hand, the background/ZDC ratio of PHENIX in Run5 and Run6, and the ratio of STAR in Run6 as well, are all similar. The ratio of STAR in Run5 is apparently larger than others, which might be relevant with the STAR background problem in Run5. 

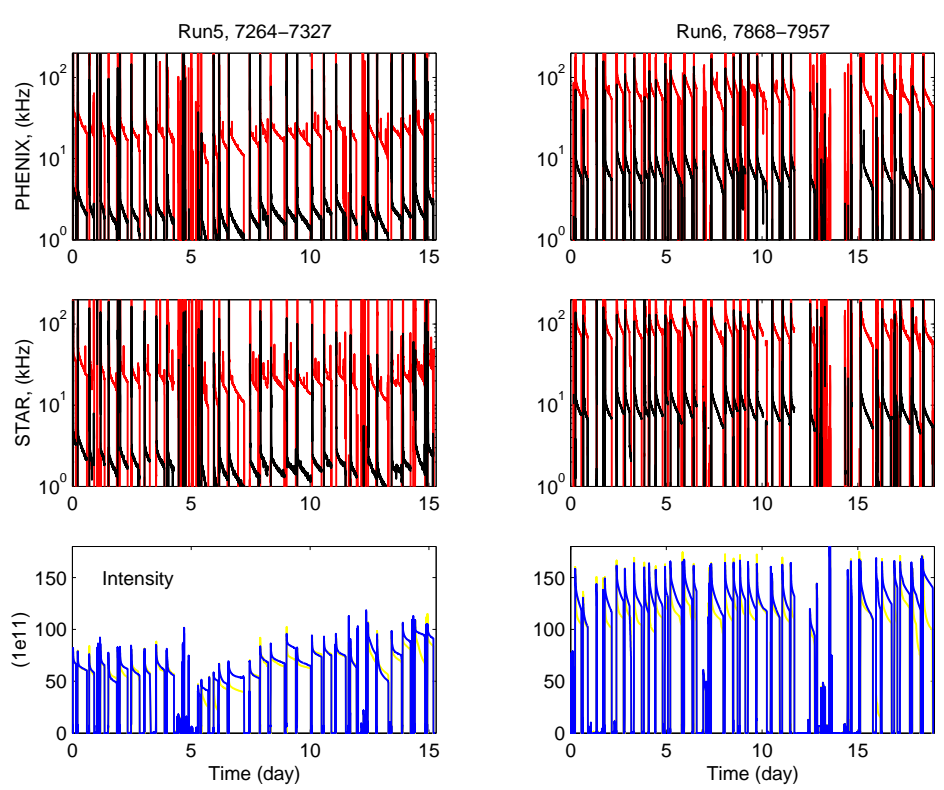

Figure 3: Beam intensity, PHENIX and STAR background and ZDC for last 30 fills in Run5 (7264-7327) and in Run6 (7868-7957).

In Fig.4, the correlations of the background and ZDC for STAR and PHENIX in Run 5 and Run6 are shown for the last 30 fills in Run5 and Run6. The background/ZDC ratio of PHENIX in Run5 and Run6, and STAR in run6 as well, are remarkably similar and close to 10 . This ratio is reduced below 10 at higher ZDC coincident rates.

The background/ZDC ratio of STAR in Run5 is 11 or higher. In the machine shutdown between Run5 and Run6, shieldings were installed between the triplets, which are usually the sources of beam-chamber-interactions, to the IR, where the detectors are located. The improvement of the background/ZDC ratio in Run6 at STAR is likely due to the shieldings.

In Fig.5, a close look of the background/ZDC ratio for typical fills in Run5 $(7325,7327)$ and Run6 $(7908,7909)$ is shown.

At the early store in Run6, the background/ZDC ratio is below 10 for both PHENIX and STAR. It seems growing in time. It is noted that the bunch length was increased more than $50 \%$ during the store for the fills in Run6, whereas it was increased very little for the fills in Run5. 

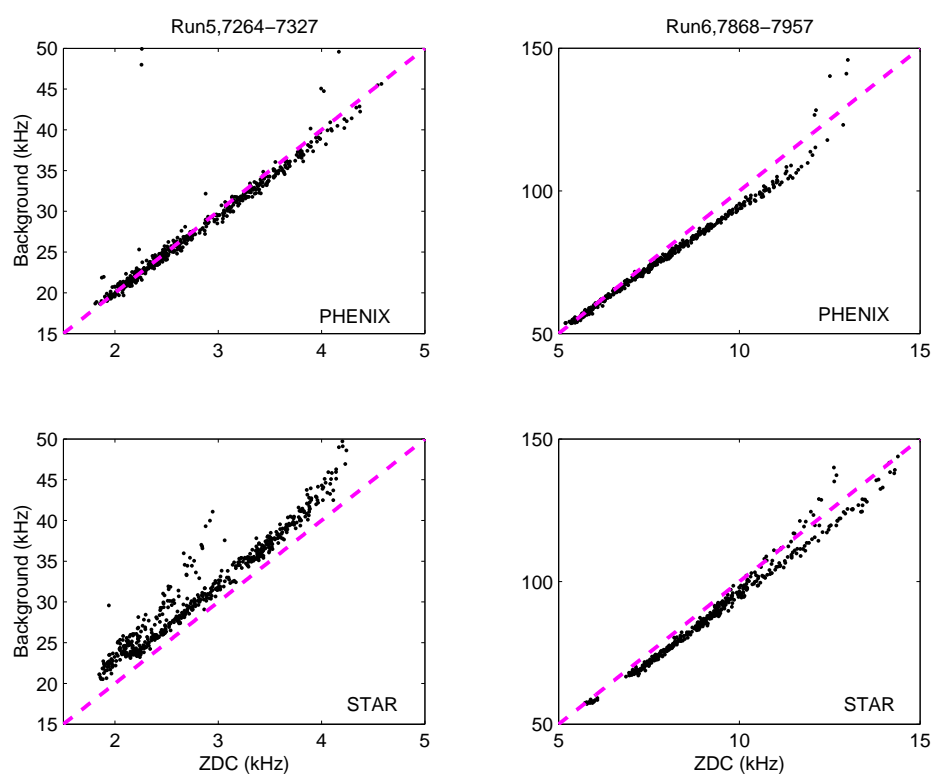

Figure 4: Correlation of the background and ZDC of PHENIX and STAR for last 30 fills in Run5 and Run6. The magenta line is for background/ZDC ratio of 10 .
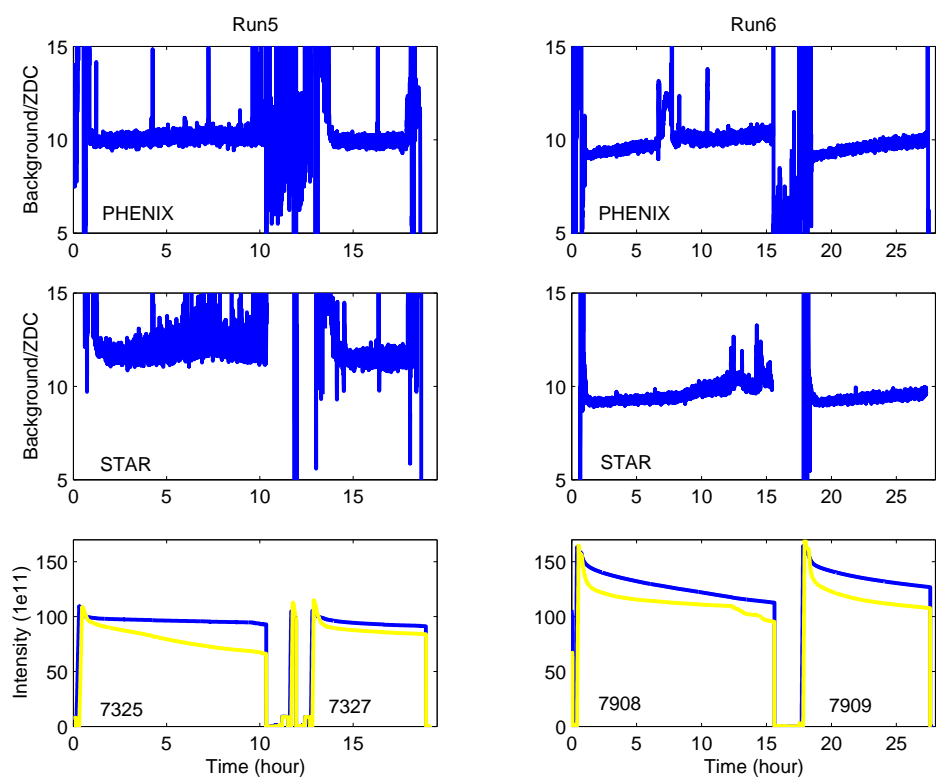

Figure 5: Background/ZDC ratio of 7325,7327 in Run5, and 7908,7909 in Run6 for PHENIX and STAR. 


\section{References}

[1] S.Y. Zhang, Experiment Background in RHIC Deuteron-Gold Run, CA/AP/107, Sep. 2003.

[2] S.Y. Zhang, Estimate of $\mathrm{Cu}-\mathrm{Cu}$ Run Experimental Background at Phobos, C-A/AP/185, Feb. 2005.

[3] S.Y. Zhang, H.C. Hseuh, C. Montag, V. Ptitsyn, D. Trbojevic, Pressure Rise in Run6 and Related Issues, C-A/AP/233, Apr. 2006. 